\title{
Subject Index Vol. 14, No. 4-6, 1994
}

Abbot Bertario 477 Age of Enlightenment 461 Alberto de’ Zancari 313 Albuminuria 365,371 Alchemy 295 Ambrosian codex 317 Anatomopathology 290 Anatomy 270 Ancient medicine 295 Anonymous medical excerpts 488 Aquatic animals 458 Arabic medicine 270 Aristotle 302 Armanni-Ebstein lesion 448 Asia 295 Atomism 337 Avicenna 270 Benedictine monasteries 259 Bible 279 Bizzozero 330 Blackall 371 Bladder lesions 494 stones 432 Blood clotting 330 pressure measurement 436 Bloody urine 494

Body homeostasis 307

Bologna 313

Bostock 365

Botanical medicines 377

Botany 259

Bright's disease 436, 443

Caseous lesion 448 Cassiodorus 477 19th century 436

- $\quad$ - Germany 443

China 295

Classical medicine 259 Codex Casinensis 69494

- $\quad 97$ from Montecassino 477

Constantinus Africanus 488

Cotugno 325

Daoism 295 Diabetes mellitus 448 Dietary salt 426 Dioscorides 259,418 Diuretics 377 Dropsy 365,371

Early medieval medicine 317 Edema 432 Edible plants 423 Empiricism 257 Endoscopy 344

Epithelium 355 Essential hypertension 436 Ethics 264

Familial hypertension 432 Favus 467 Formularia 384

Galenic medicine 317

Henle 355

Henoch-Schönlein purpura 467 Hildegarde von Bingen 391 Hippocrates 264 recommendations 494 History of hypertension 432

- medicine 257,282, 337

- nephrology 344,412,418,436

- pharmacology 391,399 Human evolution 426

Islam 270

Juvenal 477

Kidney 279

Lithiasis 290 Lithotomy 473 Lithotrity 473 Loop of Henle 355

Magic ritual 384 Maimonides 307 Marine animals 458 Mechanism 337 Medical education 257 use of salt 426

works of Galen 488 Medicinal herbs 259 
plants 399,412,418,423 Medieval medicine 478 Mercury poisoning 371 Microscopy 355 Micturition 494

Middle Ages 282,384,391 Morality 279 Mosaics 423

497

Multidisciplinarity 361 Mycology 467

Naturalism 337

Nephritis 443

Nephrolithiasis 313

Nephrology 264, 270, 290, 295, 307,

325,361 Nephrotic syndrome 325

Osmoregulation 458

Pharmacology 384 Pharmacy 384

- $\quad$ and magic 391

Platelets 330

Plenck 377

Pliny the Elder 399,412 Pompeii 423 Prognosis 264 Proteinuria 325

Ravenna School of Medicine 317

Renaissance 290

Renal anatomy 320, 344, 355, 461

disease(s) 399,412,418,448

function 302

microstructure 320

pathology 361, 371

physiology 461

structure 302

tubule 320 Rhazes 270

Salerno School of Medicine 259, 477,

478,483 Salt 426

monopolies 426

taxes 426 Schumlansky 320 Spain 290 Stroke 432 Surgery 478

Surgical instruments 473 Symbolism of salt 426

Theophilus 282

Theories of urine formation 344 Treatment of women 483 Trichophyton schönleinii 467

Tuberculosis 448

University of Bologna 361 Uraemia 365 Urinalysis 443, 452 Urinary calculi 461

disorders 461

microscopy 452

sediment 443,452

stones 494 Urine formation 302

Uroscopy 282, 307, 365, 473, 478, 483

Vascular pathology 436 Vitalism 337

Women's status 391 Works of Alcuin 488

crates 488

- Hippocrates and Pseudo-Hippo- 
Subject Index 